\title{
Multiparametric Analysis of Circulating Exosomes and Other Small Extracellular Vesicles by Advanced Imaging Flow Cytometry
}

\author{
Sotiris Mastoridis ${ }^{*}$, Giuliana Minani Bertolino ${ }^{2}$, Gavin Whitehouse ${ }^{1}$, Francesco Dazzi ${ }^{2}$, \\ Alberto Sanchez-Fueyo ${ }^{1}$ and Marc Martinez-Llordella ${ }^{1}$
}

${ }^{1}$ MRC Centre for Transplantation, Institute of Liver Studies, King's College London, London, United Kingdom, ${ }^{2}$ Regenerative Medicine, Division of Cancer Studies and Cancer Research UK King's Health Partners, King's College London, London, United Kingdom

OPEN ACCESS

Edited by:

Philippe Saas,

INSERM U1098 Interactions Hôte-Greffon-Tumeur \& Ingénierie

Cellulaire et Génique, France

Reviewed by:

Adam Bruno Ceroi,

Ghent University, Belgium

Viktoria Weber,

Danube University Krems, Austria

Johan Van Der Vlag,

Radboud University Nijmegen, Netherlands

*Correspondence:

Sotiris Mastoridis

sotiris.mastoridis@kcl.ac.uk

Specialty section:

This article was submitted

to Inflammation,

a section of the journal

Frontiers in Immunology

Received: 01 February 2018

Accepted: 26 June 2018

Published: 06 July 2018

Citation:

Mastoridis S, Bertolino GM, Whitehouse G, Dazzi F, SanchezFueyo $A$ and Martinez-Llordella $M$ (2018) Multiparametric Analysis of

Circulating Exosomes and Other

Small Extracellular Vesicles by Advanced Imaging Flow Cytometry.

Front. Immunol. 9:1583.

doi: 10.3389/fimmu.2018.01583
Extracellular vesicles (EVs) are responsible for a multitude of physiological functions, including immunomodulation. A heterogenous mixture of small EV (sEV) subsets, including putative exosomes, is derived when commonly used "exosome" isolation techniques are employed. Subset diversity relates in part to their different intracellular origins, and can be associated with distinct functional properties. Recent progress in the EV field has enabled the categorization of such subsets based on their surface composition. For the first time, we combine such emerging subset-specific markers with advanced imaging flow cytometry (iFCM) to perform high-throughput, multiparametric, vesicle-by-vesicle characterization, and functional assessment of specific small EV subsets, and exosomes in particular. The approach allows researchers to address three important applications. First, it is known that different isolation techniques result in the divergent recovery of particular vesicle subsets. Taking three commonly used "exosome" isolation techniques as test cases (ultracentrifugation, size-exclusion chromatography, and polymer-based precipitation), the capacity for convenient and accurate isolate compositional analysis by iFCM is demonstrated. The approach was able to corroborate and to quantify the known skewing of subtype recovery among different isolation approaches. Second, exosomes are a particularly widely studied EV subset. Applying exosome-specific markers to samples collected from an optimal clinical transplantation model, we verify the capacity for iFCM to detect exosomes in circulation, to establish their tissue of origin, and to provide insights as to their functional immunological potential. Finally, we describe a technique for establishing whether the transfer of a molecule of interest to a target cell is exosomally mediated. In so doing, we highlight the approach's utility in assessing the functional impact of circulating exosomes and in identifying their targets. In conclusion, we set out a new methodological approach by which small extracellular vesicle subsets, exosomes in particular, can be conveniently and comprehensively investigated, thereby offering novel phenotypic and functional insights.

Keywords: exosome, vesicle, cytometry, tetraspanin, imageStream 


\section{INTRODUCTION}

Extracellular vesicles (EVs) are nanosized membrane-bound particles released by most cell subsets. Owing to their capacity to transport a variety of biomolecules, they are key mediators of intercellular communication and as such, are the focus of increasing research interest (1-3). Much of this work has brought to light the capacity for EVs to regulate the immune system, with EVs from both immune and non-immune cells shown to be able to stimulate or suppress innate and adaptive immune responses (1, 3-5). Among their surface protein cargo, EVs can carry MHC and peptide/MHC complexes, costimulatory molecules, and adhesion molecules. The extracellular domains of these remain exposed on the vesicle surface thereby preserving their functionality, with their topology mirroring to an extent that of the parent cell (6).

As a field in its infancy, the characterization and classification of subtypes of EVs remain in flux. It is increasingly evident that diverse subpopulations of EVs secreted by different intracellular mechanisms and displaying varying functional properties exist. Such heterogeneity holds true for EVs in the $50-150 \mathrm{~nm}$ size range, often collectively referred to as exosomes, but more accurately described as "small EVs" (sEVs) - a term which is coming into more widespread use $(7,8)$. That is to say, isolates previously assumed to contain "exosomes" are now recognized to contain a mixture of exosomal and non-exosomal sEVs. Exosomes are defined in part by their small size, but also by their content of endosome-associated proteins relating to their unique endosomal biogenesis. The tetraspanin protein CD63 is particularly associated with endosome-derived exosomes. sEVs enriched in CD9 but lacking CD63 and CD81 are associated with an early endocytic signature and the plasma membrane. Finally, sEVs not associated with the endosomal pathway were devoid of all three of these tetraspanin markers (7). Further to these insights, recent observations indicate that variations in sEV tetraspanin content in fact denotes different functional properties since, as well as playing an important role in sEV biogenesis, tetraspanins regulate the routing and sorting of specific biomolecules into sEVs (9-11).

A convenient approach to vesicle-by-vesicle analysis employing such markers has, however, been lacking, making subsetspecific analyses difficult. Such analysis of individual sEVs is primarily restricted by their size. Techniques which permit their visualization, such as electron microscopy or atomic force microscopy, preclude the analysis of sEVs in large numbers, thereby limiting robust statistical assessments of heterogeneity. Proteomics, Western blotting, lipidomics, and flow cytometry of bead-captured vesicles are useful methods in the analysis of bulk isolates but are unable to distinguish variations in the number of vesicles from changes in molecular composition and are incapable of multiparametric analysis of single sEVs. Although flow cytometry is an ideal technique for high-throughput and multiparametric characterization of individual cells, most conventional flow cytometry (cFCM) platforms suffer from detection thresholds above $500 \mathrm{~nm}(12-15)$. With more than $80 \%$ of EVs reported to be $<500 \mathrm{~nm}$ in size, the majority of cFCM analyses of EVs to date have characterized only the tip of the EV iceberg, with the smallest EVs including exosomes remaining particularly elusive (16).
Advanced imaging flow cytometry (iFCM) by ImageStream ${ }^{\mathrm{x}}$ (ISx, EMD Millipore, Seattle, WA, USA) has been shown to offer significant advantages over cFCM in this regard $(14,17)$. The capacity for submicron particle and small EV (sEV) detection by iFCM has been comprehensively validated by Erdbrugger and colleagues in recent publications $(14,18)$. Here, we demonstrate a novel approach combining iFCM with subset-specific markers for the high-throughput, multiparametric, characterization, and functional assessment of circulating exosomes in particular, as well as other sEV subsets. A methodological framework for such analysis is provided, and we set out three key applications of this approach in order to assist researchers in the robust subset analysis of heterogeneous $\mathrm{sEV}$ isolates, and for the sensitive detection, phenotyping, and functional impact assessment of bona fide circulating exosomes.

\section{MATERIALS AND METHODS}

\section{Preparation of Blood Plasma}

All sample collection and preparation protocols were approved by the North of Scotland Research Ethics Committee (REC Ref: 15/NS/0062). Participants provided written informed consent. Peripheral blood was collected following standard procedures that minimize contamination by platelets and platelet-derived vesicles (19). Briefly, following cubital vein venepuncture, $3 \mathrm{~mL}$ of blood was discarded before collection of $9 \mathrm{~mL}$ into BD Vacutainer ${ }^{\circledR}$ K3-EDTA-coated collection tubes (Beckton Dickinson, USA). Tubes were inverted gently five times and blood was allowed to sit at room temperature for $30 \mathrm{~min}$. Whole blood was then centrifuged (Heraeus Megafuge 40R with $195 \mathrm{~mm} 7500-3180$ rotor, Thermo Scientific) at $400 \mathrm{~g}$ for $10 \mathrm{~min}$ at $20^{\circ} \mathrm{C}$ to remove cells. The plasma layer was collected and centrifuged again at 5,000 g for $10 \mathrm{~min}$ at $20^{\circ} \mathrm{C}$. The resulting platelet poor plasma (PPP) was aliquoted and stored at $-80^{\circ} \mathrm{C}$.

\section{sEV Isolation}

Small EVs were isolated from PPP by three commonly used "exosome" isolation techniques-ultracentrifugation (UC), size-exclusion chromatography (SEC), and polymer-based precipitation (PBP) (20-22). For UC, PPP was centrifuged (Sorval Legend Micro 21R equipped with 7500-3424 rotor, Thermo Scientific) at $10,000 \mathrm{~g}$ for $30 \mathrm{~min}$. $0.85 \mathrm{~mL}$ supernatant was resuspended in $10 \mathrm{~mL}$ of $0.22 \mu \mathrm{m}$-filtered (Merck) phosphatebuffered saline (fPBS) in ultracentrifuge tubes (Ultra-Clear Tube, Beckman Coulter), and putative exosomes (sEVs) were pelleted at $100,000 \mathrm{~g}$ for $70 \mathrm{~min}$ at $4^{\circ} \mathrm{C}$ in a SW41 Ti rotor (Beckman Coulter). The pellet was resuspended in $200 \mu \mathrm{L}$ fPBS. SEC was performed using CellGS Exo-Spin ${ }^{\mathrm{TM}}$ Mini Columns according to manufacturer's instructions. In brief, PPP was centrifuged at $16,000 \mathrm{~g}$ for $30 \mathrm{~min}$ (Sorval Legend Micro 21R equipped with 7500-3424 rotor, Thermo Scientific). Following equilibration of columns with $200 \mu \mathrm{L}$ fPBS centrifuged for $10 \mathrm{~s}$ at $50 \mathrm{~g}$ (Centrifuge 5430R, equipped with FA-45-24-11-HS rotor, Eppendorf), $0.1 \mathrm{~mL}$ PPP was applied to the column and centrifuged at $50 \mathrm{~g}$ for $60 \mathrm{~s}$. The column was then transferred to a new $1.5 \mathrm{~mL}$ collection tube, $200 \mu \mathrm{L}$ fPBS applied to the top, and elution of 
putative exosomes (sEVs) performed by a final centrifugation step at $50 \mathrm{~g}$ for $60 \mathrm{~s}$. Eluate volume was topped up to $200 \mu \mathrm{L}$ if necessary with $\mathrm{fPBS}$, aliquoted, and stored at $-80^{\circ} \mathrm{C}$. PBP was performed by ExoQuick ${ }^{\mathrm{TM}}$ (System Biosciences, Mountain View, CA, USA) according to manufacturer's instructions with minor modifications. In brief, PPP was treated with $0.5 \mathrm{U} / \mathrm{mL}$ thrombin at room temperature for $5 \mathrm{~min}$, then centrifuged at $10,000 \mathrm{~g}$ for 5 min (Sorval Legend Micro 21R equipped with 7500-3424 rotor, Thermo Scientific). The $0.25 \mathrm{~mL}$ supernatant was incubated for $30 \mathrm{~min}$ at $4^{\circ} \mathrm{C}$ with $63 \mu \mathrm{L}$ Exoquick solution, and then centrifuged at $1,500 \mathrm{~g}$ for $30 \mathrm{~min}$. The putative sEV/"exosome" pellet was then resuspended in $200 \mu \mathrm{L}$ fPBS, aliquoted, and stored at $-80^{\circ} \mathrm{C}$.

\section{Nanoparticle Tracking Analysis (NTA)}

Size distribution and concentration of isolated vesicles were measured by NanoSight NTA (LM10, Malvern Inst. Ltd., UK). The NTA analyses the motion of particles illuminated by a laser, from which it deduces their size and concentration. Samples were diluted $(1: 5,000)$ with $\mathrm{fPBS}$ and readings taken in triplicates over $30 \mathrm{~s}$, with manual monitoring of temperature and camera level set to 14. Analysis, including taking averages of triplicate readings, was performed using NTA v3.1 software, with detection threshold set to 7 .

\section{Protein Estimation and Western Blot}

Small EV preparations were diluted (1:2) in lysis buffer with final concentration of $50 \mathrm{mM}$ Tris- $\mathrm{Cl}$ (Promega, Medison, USA), $150 \mathrm{mM} \mathrm{NaCl}$ (BDH, Poole, UK), 1\% v/v Triton ${ }^{\mathrm{TM}} \mathrm{X}-100$ (BDH), $0.5 \% \mathrm{v} / \mathrm{v}$ sodium deoxycholate (Sigma-Aldrich, St. Louis, USA), $3 \% \mathrm{v} / \mathrm{v}$ sodium dodecyl sulfate (Merk, San Diego, CA, USA) and in presence of $1 \mathrm{mM}$ phenylmethylsulfonyl fluoride (Sigma-Aldrich), Complete Protease Inhibitor Cocktail (Cat \#04693124001, Roche, Mannheim, Germany), and Halt Phosphatase Inhibitor Cocktail (Cat \#04906837001, Roche). Samples were solubilized on ice for $30 \mathrm{~min}$, followed by centrifugation at $13,000 \mathrm{~g}$ for $15 \mathrm{~min}$ at $4^{\circ} \mathrm{C}$. Supernatant was collected and protein estimation was performed using Novagen BCA kit (Merk) as per manufacture's protocol. Twenty micrograms of protein lysate was diluted in $2 \times$ sample buffer [ $4 \%$ sodium dodecyl sulfate, $20 \%$ glycerol (Sigma-Aldrich), $0.004 \%$ bromphenol blue (Sigma-Aldrich), and $0.125 \mathrm{M}$ Tris-Cl $\mathrm{pH}$ 6.8] to a final volume of $30 \mu \mathrm{L}$ and loaded into $10 \%$ SDSPAGE gel in non-reducing conditions. The electrophoresis was performed with continuous buffer system and proteins were transferred by wet electroblotting onto polyvinylidene difluoride (PVDF) membranes (Bio-Rad, Hercules, CA, USA). To confirm the transfer and validate that all samples contained similar amounts of protein, the PVDF membrane was stained for 5 min with ATX Ponceau S red staining solution (Sigma-Aldrich). Membranes were blocked in 5\% non-fat milk (Bio-Rad) in Trisbuffered saline containing $0.05 \%$ Tween-20 (Sigma) for $1 \mathrm{~h}$, and then probed with the following primary antibodies: anti-CD63 (TS63, 1:1,000, Life Technologies, Carlsbad, CA, USA), anti-CD9 (M-L13, 1:250, BD Biosciences, San Diego, CA, USA), and CD81 (M38, 1:500, Life technologies) for $18 \mathrm{~h}$ at $4^{\circ} \mathrm{C}$. Membranes were incubated with anti-mouse HRP-conjugated secondary antibody (Cat \#P0260, 1:1,000, Dako, Glostrup, Denmark) for $1 \mathrm{~h}$ at room temperature. The proteins bands were visualized after incubation with Pierce ECL substrate (Thermo Scientific, Rockford, IL, USA) and development in Amersham Hyperfilm ECL (GE Healthcare, Buckinghamshire, UK).

\section{Transmission Electron Microscopy (TEM)}

Small EV preparations were placed on Formvar-coated copper grids and allowed to settle for $5 \mathrm{~min}$, without being allowed to dry. sEVs were then fixed with $2 \%$ glutaraldehyde for $5 \mathrm{~min}$ and subsequently briefly washed three times with distilled de-ionized water. After washing, the grids were stained for 20 min with 3\% uranyl acetate: $2 \%$ methyl cellulose (1:9). Imaging of sEVs was carried out using a FEI Tecnai $\mathrm{G}^{2}$ transmission electron microscope, operated at $200 \mathrm{kV}$, fitted with a Gatan Ultrascan US1000 camera.

\section{EV Labeling and Small Particle Calibrators}

Labeling was performed as previously described with minor adaptations $(17,18,23)$. The ISx requires small volumes of EV sample $(25-100 \mu \mathrm{L})$. In most instances we opted for $30 \mu \mathrm{L}$, adjusting buffer/reagent/antibody concentrations appropriately, since no wash steps are required or performed following labeling. The primary purpose of dilution is to find a balance between timely acquisition and potential complications associated with too high concentration-for instance "swarm." ISx is less likely to suffer from such coincident detection than cFCM platforms, particularly if sample concentrations $<10^{10}$ events $/ \mathrm{mL}$ are used. $3 \mu \mathrm{L}$ PPP, SEC, and PBP or $4.8 \mu \mathrm{L}$ UC samples were topped up to $22 \mu \mathrm{L}$ fPBS, and $0.5 \mu \mathrm{L}$ Fc receptor blocker (Human TruStain $\mathrm{FcX}^{\mathrm{TM}}$, BioLegend) was added for $10 \mathrm{~min}$ at room temperature. 5-(and-6-)-carboxyfluorescein diacetate succinimidyl ester (CFDA-SE, ThermoFisher) was added to the EV-PBS solution to give a final running concentration of $10 \mu \mathrm{M}(2.5 \mu \mathrm{L}$ volume), allowed to incubate in the dark for $10 \mathrm{~min}$ at $4^{\circ} \mathrm{C}$, and followed by further staining in the dark at room temperature for $15 \mathrm{~min}$ with master-mix preparations in fPBS ( $5 \mu \mathrm{L}$ volume) of the following monoclonal antibodies (mAbs) as appropriate: anti-human HLAA3-APC (eBioscience, GAP.A3); CD9-PE (BioLegend, HI9a); anti-human CD63-PE (BioLegend, H5C6); CD81-PE (BioLegend, 5A6); HLADR-PECY7 (Biolegend, L243), HLAB8-APC (Miltenyi, REA145), HLAB7 (Miltenyi, REA176); PD-L1-BV605 (Biolegend, 29E.2A3); IgG1 (BioLegend, MOPC-21); and IgG2b (BioLegend, MPC-11). All mAbs were centrifuged at 5,000 g for $5 \mathrm{~min}$ prior to use, as clumps could be mistaken for EVs $(13,18,24)$. CFDA-SE is cleaved of acetate groups by EV esterases and converted to CFSE (carboxyfluorescein succiminidyl ester), which serves as a fluorescent pan-EV label. Optimized protocols for the labeling of EVs by such dyes have been published, and are preferred for their relative ease, specificity, and the circumvention of wash steps $(18,23,24)$. To avoid false positive events, all antibodies used were run on ISx in buffer (fPBS) alone to ensure antibody clumps were not present. Verification of small particle detection was performed using fluorescently labeled submicron polystyrene beads (PSB) and liposomes. ApogeeMix (Apogee Flow Systems, UK) contained green beads 110 and $500 \mathrm{~nm}$ in size. Liposomes were prepared as previously described, with CFTM-labeling performed by the addition of 1\% 1,2-dioleoyl-snglycero-3-phosphoethanolamine-CFTM488 (CF-DOPE) to the 
lipid film (25). EV lysis was performed by incubating fPBS-diluted EVs in $0.1 \%$ Triton $^{\mathrm{TM}} \mathrm{X}-100$ (Thermo Scientific) for $15 \mathrm{~min}$ at room temperature.

\section{ImageStream ${ }^{x}$ Small Particle Acquisition and Analysis}

Multispectral imaging flow-cytometric acquisition of EVs and small particle calibrators was performed using Amins ImageStream $^{\mathrm{x}}$ MKII (ISx, EMD Millipore, Seattle, WA, USA) with fluidics set at low speed, sensitivity set to high, magnification at $60 \times$, core size $7 \mu \mathrm{m}$, and the "Hide Beads" option unchecked prior to every acquisition in order to visualize speed beads in analyses (Figure S1 in Supplementary Material). All parameters are stored in acquisition template except the latter, which requires unchecking prior to each acquisition. The ISx was equipped with the following lasers run at maximal power to ensure maximal sensitivity: $405 \mathrm{~nm}(120 \mathrm{~mW}), 488 \mathrm{~nm}(200 \mathrm{~mW}), 561 \mathrm{~nm}$ $(200 \mathrm{~mW})$, and $642 \mathrm{~nm}(150 \mathrm{~mW})$. Upon each startup, the instrument calibration tool ASSIST ${ }^{\circledR}$ was performed to optimize performance and consistency. Each of the two charged couple device (CCD) cameras with which the ISx is equipped have six channels of detection. Two channels (Ch01 and Ch09) were set to brightfield (BF), permitting spatial coordination between cameras. Channel 12 was set to side-scatter (SSC), and further fluorescence channels were used for antibody detection as required (Figure S1 in Supplementary Material). The advanced fluidic control of ISx, coupled with the presence of continuously running speed beads enable cell/particle enumeration using the "objects per $\mathrm{mL}$ " feature within the IDEAS ${ }^{\circledR}$ data analysis software. To avoid the risk of coincident particle detection, EV samples were not run at concentrations greater than $10^{10}$ objects/mL (18). All samples were acquired using INSPIRE $^{\circledR}$ software, with a minimum of $5,000 \mathrm{G} 1$ events collected, or as dictated by the type of analysis to be undertaken. Data analyses were performed, and spectral compensation matrices produced, using ISx Data Exploration and Analysis Software (IDEAS ${ }^{\circledR}$ ) (Figure S2 in Supplementary Material). Technical controls and isotype controls, in conjunction with fluorescence minus one (FMO) controls, were employed where appropriate for EV gating.

\section{sEV Uptake Assay and ImageStream ${ }^{\mathrm{x}}$ Analysis}

Peripheral blood mononuclear cell isolation was performed in PBS-diluted (1:2) fresh blood collected in 3-4 $6 \mathrm{~mL}$ collection tubes (Vacuette ${ }^{\circledR}, 456088$ ) by Ficoll-Paque (GE Healthcare, Sweden) density-gradient centrifugation. Monocytes were separated by immunomagnetic beads coated with anti-CD14 antibodies according to EasySep ${ }^{\mathrm{TM}}$ manufacturer's instructions (EasySep $^{\mathrm{TM}}$, StemCell Technologies), yielding purities $>90 \%$ as assessed by cFCM (Figure S3A in Supplementary Material) using (Heraeus Megafuge 40R with $195 \mathrm{~mm}$ 7500-3180 rotor, Thermo Scientific) at $800 \mathrm{~g}$ for $20 \mathrm{~min}$ with brake set to setting 3 . Monocytes were cultured in 96-well plate (200,000/well) in serum-free media (X-VIVOTM 15, Lonza), with or without PBP-derived sEV added on a volume/volume basis to mirror physiologic conditions and circumvent errors intrinsic to $\mathrm{sEV}$ protein estimation (9). PBP isolation was performed on $500 \mu \mathrm{L}$ PPP according to manufacturer's instructions, with the final sEV pellet resuspended in $250 \mu \mathrm{L}$ fPBS. $500 \mu \mathrm{lPP}$ is derived from approximately $1 \mathrm{~mL}$ fresh blood, and since each culture condition was performed in $200 \mu \mathrm{L}$, a fifth of the PBP isolate was utilized in each test condition. Summarily, monocytes were cultured either

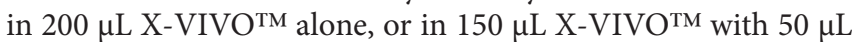
PBP isolate. Following incubation, adherent cells were collected by gentle pipetting following washing with ice cold PPBS and allowing to stand on ice with cold fPBS for $5 \mathrm{~min}$. Cells were then labeled with the following antibodies: CD45-V450 (BD, H130); CD63-PECy7 (BioLegend, H5C6); HLA-A3-APC (eBioscience, GAP.A3); and LIVE/DEAD ${ }^{\text {TM}}$-FITC (ThermoFisher Scientific). Whole-cell image acquisition was performed with fluidics set at low speed, sensitivity set high, magnification at $60 \times$, and SSC and $\mathrm{BF}$ channels set as above. Live cells were identified after sequential gating for (i) single cells, using BF aspect ratio Vs. area feature gating; (ii) in focus cells, using $\mathrm{BF}$ gradient root mean square

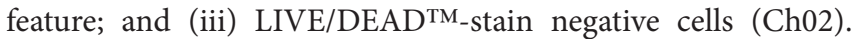
Colocalization analyses were performed on further sub-gated HLA-A3-stain positive (Ch11) populations.

The two fundamental principles needed to understand the data analysis framework of IDEAS ${ }^{\circledR}$ are Masks and Features (Figure S2 in Supplementary Material). Masks are used to spatially discriminate the area of the cell that is of interest and exclude those parts that are not. They can be created based on brightfield (BF), scatter (SSC), or fluorescence images. For instance, the morphology mask might be used for defining the nucleus, the spot mask for defining labeled cellular components by the identifying bright or dark regions, and so on. There are in the region of 20 masks available, and these can be combined in a Boolean manner to create multiple potential applications. Mask validation should always be performed by visually inspecting the mask on numerous acquired cells, and alternative approaches can often be found. Features on the other hand, are a computational algorithm able to analyze masks as their input. For instance, the quantitative assessment of internalization might be achieved by combining a spot mask from one detection channel with a morphology mask from another. The gamut of application potential and protocol descriptions have been comprehensively outlined elsewhere $(26,27)$. The approach to protein colocalization by ISx and its software package IDEAS ${ }^{\circledR}$ is well described (28-30). In summary, ISx enables quantitative analysis of the degree of colocalization between fluorophores on a pixel-by-pixel basis by comparing digital images captured in each of its image detection channels. The Similarity Bright Detail R3 algorithm within IDEAS ${ }^{\circledR}$ produces a score (SBDS) serving as a measure of the degree of colocalization between these. Comparison of the SBDS between markers of interest (in this case HLA and CD63) to the SBDS with a marker known to be diffusely expressed on all cells of interest (in this case CD45) permits SBDS interpretation (30). Quantification of fluorescent foci using is achieved using the "Spot Count" feature. A Threshold mask with adaptive erosion coefficient of 70 was applied to Channel 11 (HLA-A3) and served as the input for the "Spot Count" feature. This approach offered optimal spot identification sensitivity as confirmed by visual interrogation (although "Spot" masks or the "Spot Wizard" would be recommended in instances where 
spots might be less readily differentiated from background or for inexperienced users-see Figure S3 in Supplementary Material).

\section{Statistical Analysis}

Unless otherwise stated, statistical analyses were performed by GraphPad Prism v7.0 Software. Student's $t$-test was used for comparisons between two groups and ANOVA to compare more than two groups $\left({ }^{\star} P<0.05\right.$, ${ }^{* *} P<0.01$, ${ }^{* *} P<0.001$, and $\left.{ }^{* * * *} P<0.0001\right)$.

\section{RESULTS}

\section{Submicron Particle Detection and Multiparametric Characterization of Circulating sEVs}

The known capacity for submicron particle detection by iFCM was confirmed using fluorescent PSB 110 and $500 \mathrm{~nm}$ in size, with those of smallest size being readily resolved (Figure 1Ai). Manufactured beads are known to have higher refractive index (RI) than do biological vesicles of equivalent size $(18,31,32)$. For this reason, gating strategy was also guided using fluorescently labeled liposomes of exosomal size $(129.3 \pm 2.4 \mathrm{~nm})$ (Figure 1 Aii; Figure S3B in Supplementary Material), which more closely resemble the RI and scatter characteristics of CFDA-SE-labeled sEVs (Figure 1Aiii). CFDA-SE serves as an intravesicular dye, becoming converted to CFSE once cleaved of acetate groups by esterases present in sEVs. Following detergent lysis of sEV isolates $\left(0.1 \%\right.$ Triton $\left.^{\mathrm{TM}} \mathrm{X}-100\right)$, CFDA-SE labeling does not result in the detection of CFDA-SE-positive events, thereby confirming the requirement for vesicles to be intact for labeling to occur (Figure 1Aiv). The acquisition of appropriate control samples must be performed prior to each acquisition run, if common pitfalls associated with EV flow-cytometric profiling are to be avoided. Namely, this will help to avoid running contaminated reagents or antibodies which can lead to the acquisition of false particles. Representative ISx dot-plots of buffer alone (filtered phosphate-buffered saline), unstained EVs, and buffer plus reagents without EVs are shown (Figure 1B) and serve to reassure that none have been contaminated or formed aggregates for instance. In addition to the assessment of the scatter characteristics of fluorescently labeled particles of known size, gating of sEV populations is confirmed by single-event visual interrogation. While CFDA-SE-labeled sEVs appear as low-scatter and low/mid fluorescence intensity events, contaminating cells, EV clusters, and large EVs appear as higher scatter/intensity $(14,17,18,33)$. The difference in properties of the two CFDA-SE-positive populations (G1 and G2) is clear by visual interrogation of detected events, showing G2 to be predominantly composed of cellular debris or particle aggregates as opposed to the uniform particles in G1-the "small EV" gate (Figure 1C) (18). Multiparametric phenotyping of gated sEV (G1) was demonstrated by fluorescent labeling with EV markers, i.e., the tetraspanins CD9, CD63, CD81 (PE, Ch03), and HLA-DR (PECy7, Ch06). HLA-DR was chosen primarily for its ubiquity (Figure 1D). Appropriate isotype controls and FMO controls should be used for the setting of gates and interpretation of data. The importance of testing buffer with all antibodies and reagents to ensure that fluorescent particles with similar scatter to EVs are not produced leading to false events in G1 cannot be overstated (Figure 1D; Figure S4 in Supplementary Material).

\section{sEV Subtype Analysis and the Impact of Exosome Isolation Technique: Application 1}

To demonstrate the capacity and utility of iFCM in sEV subtype analysis, isolates from three commonly used "exosome" isolation techniques were compared. The successful isolation of exosomes from the plasma of five healthy volunteers by three commonly utilized techniques - ultracentrifugation (UC), SEC, and PBPwas confirmed by TEM, NTA, and Western blot for canonical exosomal markers (Figures 2A-E; Figure S5 in Supplementary Material). The size profile of isolated vesicles, as determined by NTA, was equivalent across isolation approaches. Estimation of the concentration of sEV s by NTA shows UC to isolate significantly fewer particles per $\mathrm{mL}$ plasma than SEC or PBP (Figure 2D) in keeping with previous reports $(22,34,35)$.

Analysis of the Scatter/CFDA-SE profiles of samples revealed that as a percentage of all detected non-speed bead events sEVs were lowest in PPP, thereby confirming successful sEV enrichment by all isolation methods tested (Figures 2F,Gii). The greatest enrichment or "purification" of sEV was seen in UC isolated samples (Figures 2F,Gii). However, the recovery of sEVs from plasma was significantly less in the case of UC compared to other methods, as quantified by the "objects per $\mathrm{mL}$ " function (Figure 2Gi). PPP was noted to contain relatively high proportions of CFDA-SE-negative particles displaying varying degrees of Scatter (G3), but also of CFDA-SE-positive particles with high Scatter than sEVs (G2), likely to represent debris, EV clusters, large EVs, or apoptotic bodies. SEC and PBP samples also exhibited relatively higher proportions of high-Scatter events, thereby contributing to their lower sEV enrichment estimation as compared to UC (Figures 2F,G). sEV subset analysis was performed by assessing the number and proportions of gated sEVs (G1) bearing subset markers. Again, all three methods achieved enrichment from PPP starting material of bona fide exosomes-defined as G1-gated CD63+ events (Figure 2Giv). However, the recovery of bona fide exosomes, assessed as the number of CD63 + sEV "objects per $\mathrm{mL}$ ", was also shown to be lowest following UC (Figure 2Giii). CD9 was observed to be the most commonly contained tetraspanin in sEVs across all samples, while CD81 was noted to be particularly enriched in UC isolates (Figure 2Gv). Taken together, iFCM analysis confirmed that the recovery and the purity of sEV subsets, as defined by their content of subset-specific markers, vary among isolation techniques.

\section{Profiling of Circulating Exosomes for Tissue-Specific Biomarker Discovery and Functional Analysis: Application 2}

The utility of exosomes as biomarkers has been extolled due to their stability and ubiquity in biofluids, their capacity to offer insights into the cell or compartment of origin, and for 

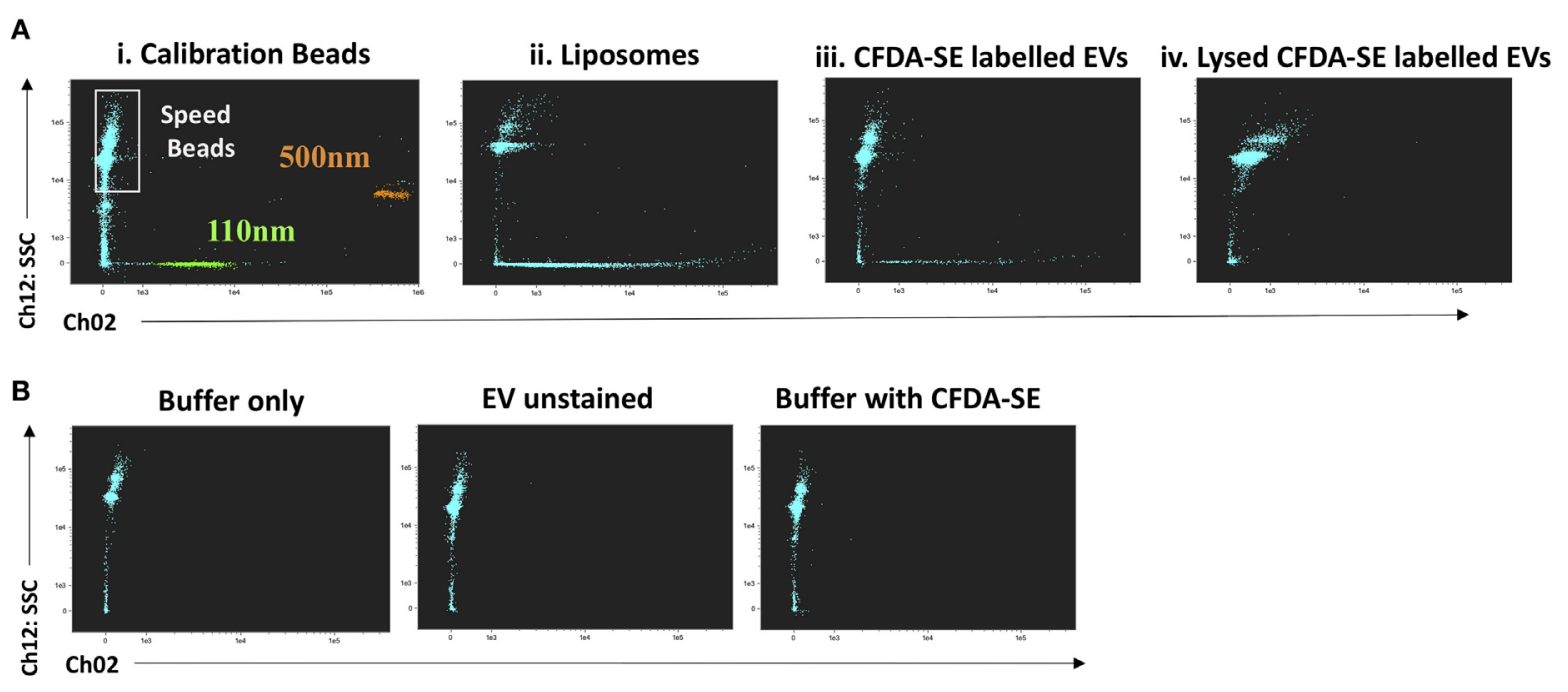

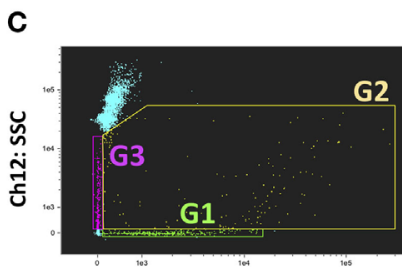

Ch02: CFDA-SE
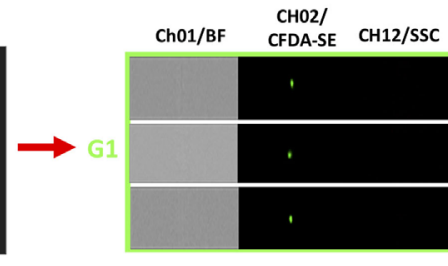

CFDA-SE POSITIVE LOW SCATTER

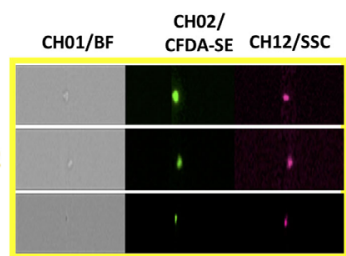

CFDA-SE POSITIVE HIGH SCATTER

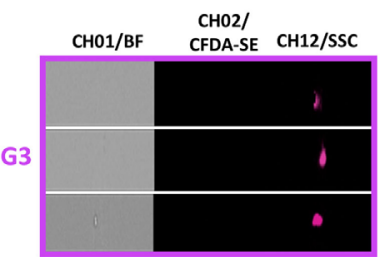

CFDA-SE NEGATIVE HIGH SCATTER

D CFDA-SE labelled EV isolate Buffer, reagents, antibodies

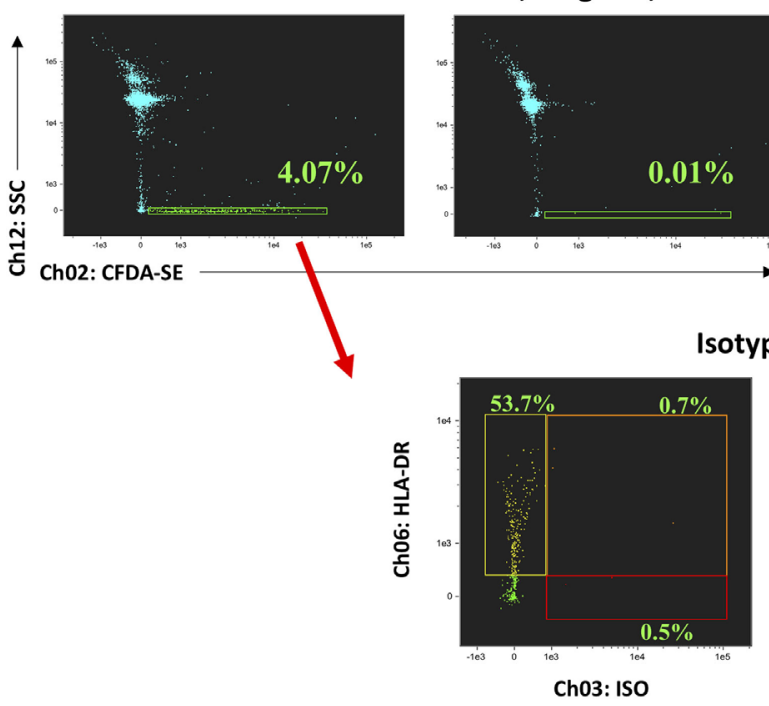

sotype controls

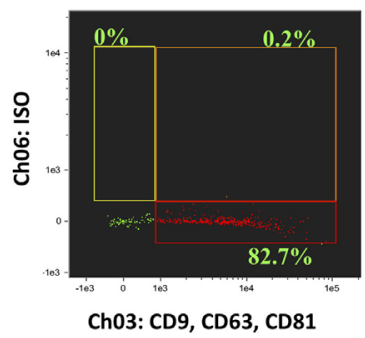

Multiparametric EV labelling

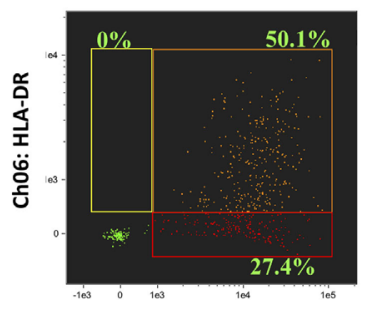

Ch03: CD9, CD63, CD81

FIGURE 1 | Submicron particle detection and multiparametric characterization of circulating "small EV" (sEV) by ImageStream (ISx). Gating sEV populations is achieved by assessment of the Scatter intensity of particles of known size, and through confirmation by single-event visual interrogation. (A) Fluorescently labeled polystyrene beads and liposomes which have a refractive index closer to that of EV are used and contrasted to CFDA-SE labeled circulating human extracellular vesicles (EVs) derived by ultracentrifugation. CFDA-SE-mediated labeling of intact vesicles is demonstrated by detergent lysis (0.1\% TritonTM X-100) of UC-derived EVs prior to labeling. (B) The acquisition of appropriate control samples is an important step prior to running experimental samples if the common pitfalls of flow-cytometric profiling of EVs are to be avoided. Representative ISx dot-plots of buffer alone (filtered phosphate-buffered saline), unstained EVs, and buffer plus reagents without EVs are shown. (C) Representative dot-plot of CFDA-SE Vs. Scatter intensity to demonstrate the heterogeneity of acquired events and the principle of visual interrogation in gate-setting. The difference in properties of the two CFDA-SE-positive populations (G1 and G2) is clear by visual interrogation, showing G2 to be predominantly composed of cellular debris or particle aggregates as opposed to the uniform particles in G1 -the sEV gate. (D) Multiparametric phenotyping of these gated sEV (G1) is demonstrated by fluorescent labeling with EV markers, the tetraspanins CD9, CD63, and CD81 combined (PE, Ch03) and HLA-DR (PECy7, Ch06). Fluorescence minus one and isotype controls are used to set gating. A buffer + reagent + antibody control should be performed and is demonstrated here. 

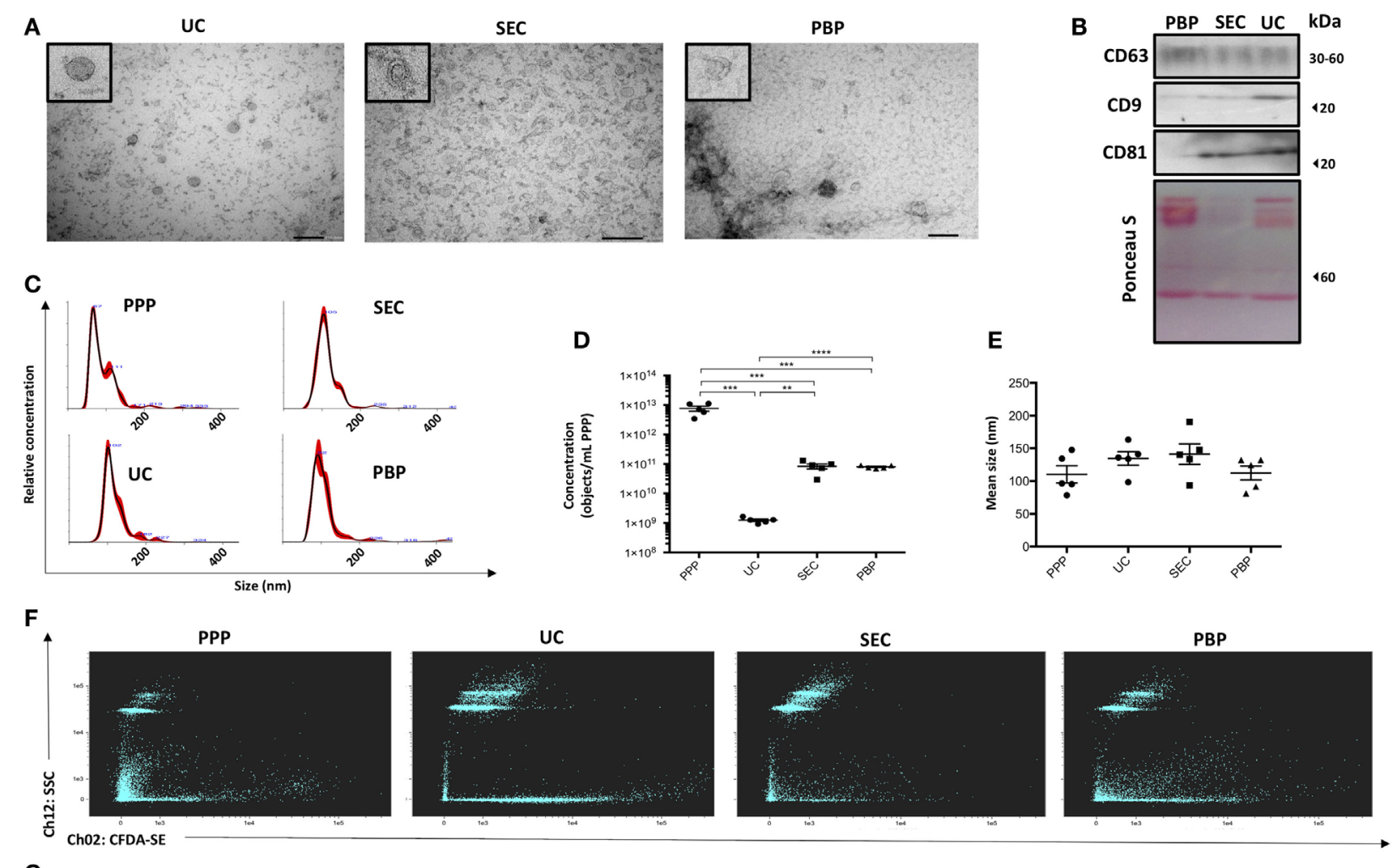

G
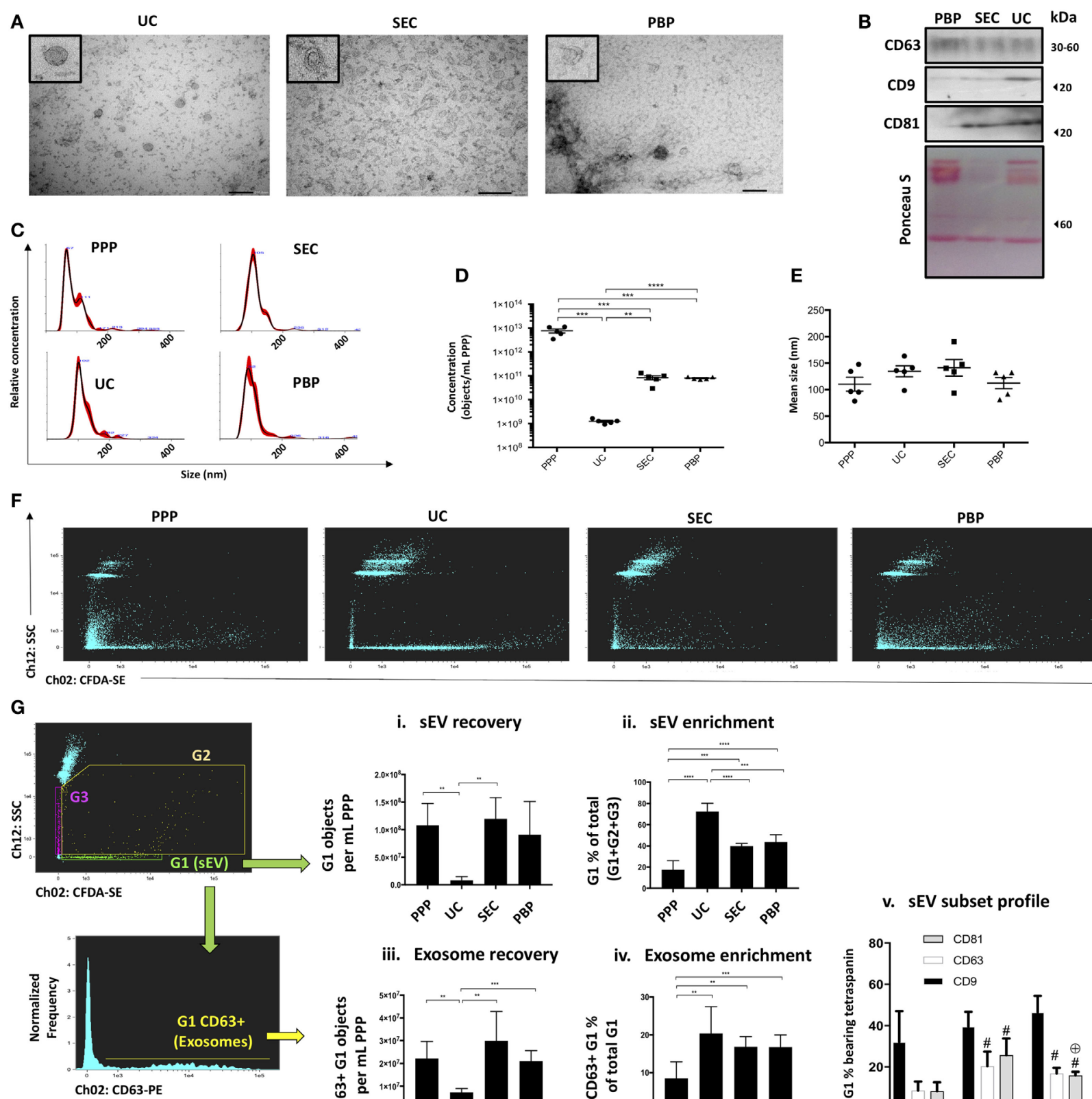

iii. Exosome recovery

iv. Exosome enrichment
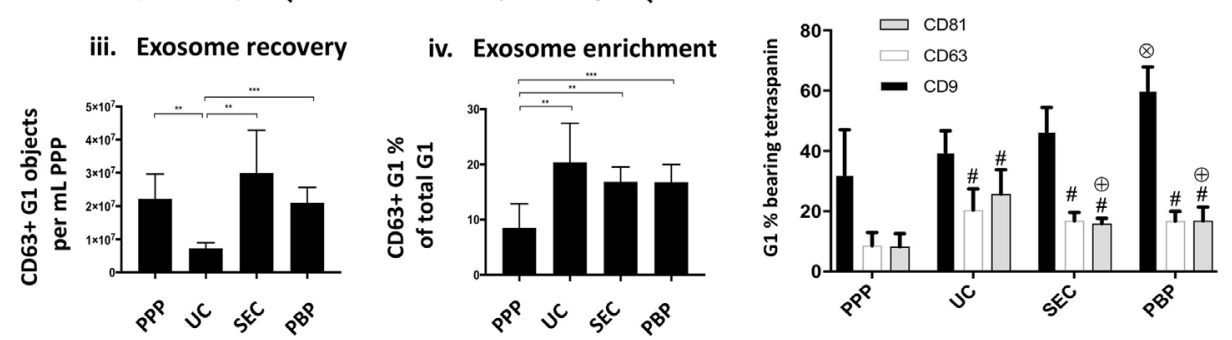

FIGURE 2 | Extracellular vesicle subtype analysis and the impact of exosome isolation technique (Application 1). Confirmation of the presence of exosomes in "small EV" (sEV) isolates from three commonly used isolation techniques (UC, ultracentrifugation; SEC, size-exclusion chromatography; PBP, polymer-based precipitation) was achieved using three methods. (A) Transmission electron microscopy images showing characteristic morphologic features of exosomes (size bars: $200 \mathrm{~nm}$ ). Single extracellular vesicles (EVs) are shown in expanded (zoomed) box in top left of each image. (B) Western blot showing canonical exosomal markers, such as CD9, CD63, and CD81. (C-E) Nanoparticle tracking analysis (NTA) profiling of isolates and of platelet poor plasma (PPP) showing size distribution and concentration profiles given "per $\mathrm{mL}$ of starting PPP" following correction for dilution/concentration steps were performed. (F) ISx dot-plot showing Scatter and CFDA-SE intensity for all methods of isolation. (G) ISx analysis of sEV subset recovery, including bona fide, CD63-positive, and exosomes. (i) sEV recovery is estimated by applying the objects per $\mathrm{mL}$ statistical feature in IDEASTM to the G1 gate. (ii) The proportion of sEVs (G1) in relation to non-speed-bead events (G2 + G3) is used as an indication of SEV enrichment achieved by UC, SEC, and polymer-based precipitation (PBP) and compared to PPP. (iii) Exosome recovery is estimated by applying the objects per $\mathrm{mL}$ statistical feature in IDEASTM to the CD63+ events within the G1 gate. In the cases of UC, SEC, and PBP, objects per mL relate to starting PPP sample, which is to say that the raw readout from the ISx "objects per $\mathrm{mL}$ " feature is corrected for any dilution/concentration steps performed. (iv) The proportion of exosomes (G1-gated CD63+ events) in relation to non-speed-bead events (G2 + G3) is used as an indication of exosome-specific enrichment achieved by UC, SEC, and PBP and compared to PPP. (v) Profiling of other canonical exosome markers CD9 and CD81, in comparison to bona fide exosome marker CD63. For consistency, representative data and images in panels $(\mathbf{A}-\mathbf{C}, \mathbf{F})$ are from a single healthy volunteer, with panel $\mathbf{( G )}$ representative plots being UC-derived. Data

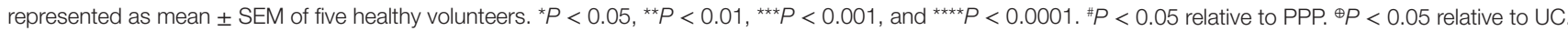
${ }^{\otimes} P<0.05$ relative to PPP, UC, and SEC. 
their selectively enriched cargo which offers an understanding of their functional impact upon cellular targets. For these reasons, a convenient, sensitive, and robust approach to their characterization from circulating blood would be of significant benefit.

To demonstrate circulating exosome detection and characterization by iFCM, the setting of clinical organ transplantation offered an optimal scenario. It provided the opportunity to combine the use of an exosome-specific marker (CD63), with an origin-specific marker (donor-HLA). In addition, the setting enabled the detection of a relatively rare population of tissuespecific exosomes with the same patient serving as the control subject (pre-transplantation) so as to rule out non-specific antibody targeting. Finally, additional markers allowed functional assessments and comparisons to be made between donor- and recipient-origin exosomes.

Bona fide CD63-positive exosomes were analyzed for their expression of donor and recipient HLA (Figure 3A). sEVs from an HLA-B27-positive, HLA-B8-negative recipient were gated in G1 before and after receiving a liver allograft from an HLAB8-positive, HLA-B27-negative donor. Donor HLA-bearing exosomes were noted to be detectable in circulation after liver transplantation. These results were further substantiated by comparing healthy volunteers of distinct HLA genotype, as well as additional liver transplant recipients (Figures 3C,D). Multiparametric iFCM analysis enabled functional comparisons of exosome populations. It has been suggested that programmed death-ligand 1 (PD-L1) released from allografts in a mouse model of liver transplantation are responsible for $\mathrm{T}$ cell exhaustion and tolerogenesis (36). Therefore, we sought to assess the differential distribution of PD-L1 in circulating exosomes of transplanted patients. Interestingly, the presence of PD-L1 was seen to be higher on donor-origin liver-derived exosomes (Figure 3B). This approach to circulating plasma-derived exosome characterization permits convenient origin identification and functional assessment to be performed in a variety of contexts.

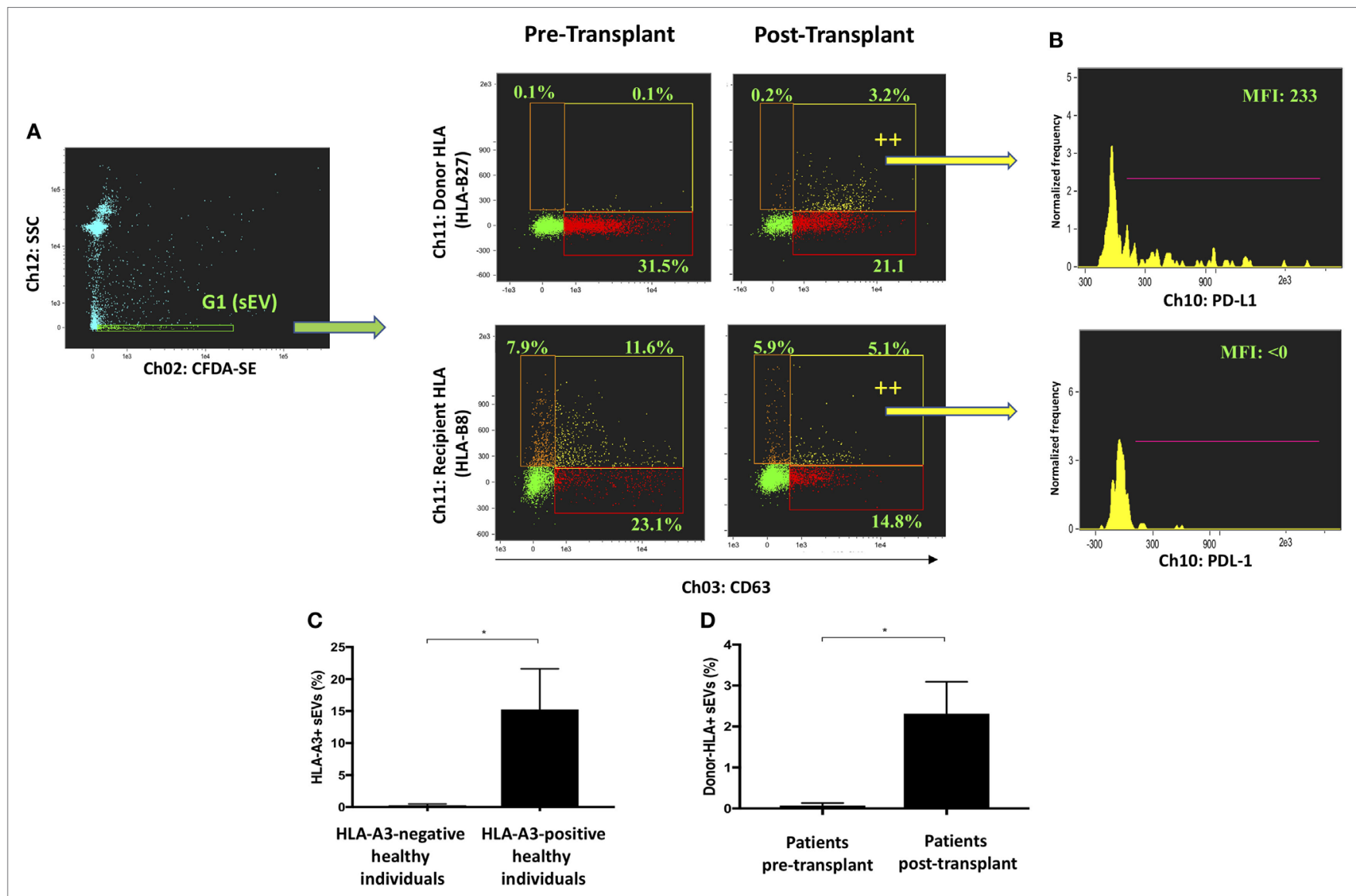

FIGURE 3 | Profiling of circulating exosomes for tissue-specific biomarker discovery and functional analysis (Application 2). (A) Small EVs (sEVs) from an HLA-B27positive, HLA-B8-negative recipient were gated in G1 before and after receiving a liver allograft from an HLA-B8-positive, HLA-B27-negative donor. Bona fide CD63-positive exosomes were analyzed for their expression of donor and recipient HLA. Donor HLA-bearing exosomes become detectable in circulation after liver transplantation. (B) PD-L1 expression was analyzed in exosomes bearing either donor or recipient HLA and found higher in the former group post-transplantation.

(C) Percentage of HLA-A3 positive sEVs were compared between genotypically confirmed HLA-A3+ $(n=3)$ and HLA-A3 negative $(n=3)$ individuals.

(D) Percentage of donor-HLA + sEVs observed pre- and post-liver transplantation, in genotypically confirmed HLA-mismatched liver transplant recipients (n3). Data represented as mean \pm SD. ${ }^{*} P<0.05$. 


\section{Target Cell Analysis Determines Exosome-Mediated Molecular Transfer: Application 3}

Establishing definitively whether molecules found on a cell's surface were exosomally transferred is difficult in clinical contexts where pre-labeling of exosomes is not possible. Exosome-mediated transfer might, however, be inferred by assessing whether the molecule of interest is colocalized with exosome-specific proteins such as CD63, since at the time of incorporation to the target cell the exosome leaves a patch of the proteins it transferred. To this end, monocytes from an HLA-A3 negative individual were incubated in vitro with sEVs from HLA-A3 positive and HLA-A3 negative (control) individuals. The colocalization of exosomal marker CD63 with HLA-A3 was assessed using the Bright Detail Similarity Score (SBDS) within IDEAS ${ }^{\circledR}$, as described previously. CD63 was chosen because it defines bona fide exosomes, but also because in most cells it is present predominantly in intracellular compartments rather than the cell surface (37). Following $2 \mathrm{~h}$ incubation of monocytes with sEVs derived from an HLA-A3 donor, the proportion of HLA-A3 positive monocytes increased significantly and continued to rise to $24 \mathrm{~h}$ (Figure 4A). SBDS for HLA-A3 and CD63 colocalization was significantly higher than among the CD45/CD63 comparator, with CD45 ("leukocyte common antigen") serving as a total-surface stain (SBDS $2.26 \pm 0.21$ Vs. $1.12 \pm 0.11 ; P<0.01$; Figure 4B). HLA-A3 fluorescence was confirmed to colocalize with CD63 by visual interrogation of high SBDS events (Figure 4C). It should be noted that not all HLA-A3 "spots" were associated with CD63; likely a reflection of our data showing that not all sEVs are CD63 containing. Following $24 \mathrm{~h}$ incubation, and without further addition of sEVs, IS $x$ image interrogation also provided clues as to the kinetics of HLA/exosome uptake. At this time point, HLA-A3 positive monocytes were either studded with a multitude of HLA-A3 foci, and/or exhibited diffuse fluorescence in the HLA-A3 channel (Figure 4C). These kinetic properties could be quantitated using the "spot count" feature in IDEAS ${ }^{\circledR}$, as described above, with data showing clear trends toward either no spots (diffuse fluorescence) or multiple spots $(>11)$ among HLA-A3 positive monocytes undergoing a longer incubation period (Figures 4D,E). So, spatial interrogation of acquired cells using ISx and IDEAS ${ }^{\circledR}$ reveals that exosome-mediated transfer of allo-HLA to CD14+ cells occurs rapidly, with HLA initially limited to the area of exosome binding followed by more generalized dispersal.

\section{DISCUSSION}

In the present report, we address the need for a convenient method by which high-throughput multiparametric phenotypic and functional analyses of sEV subsets, in particular exosomes, can be performed.

The superiority of iFCM over cFCM in the detection of submicron particles has been previously described $(14,18)$. ISx utilization of spatially registered CCD cameras, rather than photomultiplier tubes, allows for superior fluorophore detection sensitivity. While machine noise plagues CFCM sensitivity and necessitates a triggering threshold to be set, the ISx CCD does not have to exceed a threshold value above which a particle can be considered a data point. Instead, it acquires all images that are at least one pixel above the background of the camera $(14,17,38)$. The ISx calibration software, run upon every startup, performs pixel gain corrections and dark current offsetting for each one of the CCDs pixels, thereby further enhancing the detection of faint signals and providing a uniform background level (38). In practical terms, the result is that ISx is able to detect and resolve $20 \mathrm{~nm}$ fluorescent latex beads which are entirely undetectable by cFCM (17). Importantly, unlike cFCM, ISx also suffers less from swarm, with coincident detection leading to significant underestimation of counted populations. Finally, unlike many reports of EV characterization by $\mathrm{CFCM}$, there is also no requirement for specially adapted cytometers or an experienced operator capable of manual hardware adjustments and calibrations $(24,39)$. "Dedicated" small particle cytometry (dFCM) platforms such as these meet many of the requirements for sEV analysis, including high powered lasers, a stable velocity core stream, and higher sensitivity detectors (40). However, to date, they do not offer the convenience of use required by many researchers. Furthermore, cFCM (and dFCM) is low in information content, measuring only one feature per fluorescence marker (integrated intensity); compared to iFCM which allows thousands of spatial and morphological properties of individual events to be assessed-thereby permitting analyses such as those outlined in "Application 3."

Drawing on their comprehensive proteomic analyses of EVs derived from human dendritic cells, Théry and colleagues set out a sub-classification of sEVs according to tetraspanin markers (7). Different approaches to exosome isolation are known to skew the recovery of subsets toward more or fewer vesicles bearing one or another of these markers, and so the functional properties of isolates derived by different techniques are known to vary $(7,22,41)$. Indeed, this might go some way toward explaining apparent contradictions reported across published studies of EV functional analyses $(2,7)$. That is to say, since variations exist in isolation procedures among groups, it must be expected that the functional properties of isolates also vary. Such "skewing" of EV isolate is not only restricted to the specific isolation technique used, with variations in the steps involved in the preparation of plasma also having an impact. These range from venepuncture technique, the choice of blood collection tube, the length of incubation time, the means of transportation, the storage temperature, the centrifugation approach, and the number of freeze thaw cycles, to name but a few $(8,42)$. Of course, much emphasis has been given to the need for standardization of EV isolation (19). But, it comes as little surprise, that calls have been made by researchers leading the field for improved techniques enabling the accurate characterization of different populations of sEVs, including exosomes $(2,10,43)$. We show how iFCM analysis of sEV isolates offers a convenient, accurate means by which this can be achieved.

In our analysis we confirm that commonly used "exosome" isolation techniques produce differing isolates. The focus of the interpretation of this effect is not so much on making a suggestion about the relative superiority/inferiority of a particular isolation method. As mentioned, the exact outcomes of such analysis would likely vary with small alterations in protocol, between different investigated patients, across the spectrum of biofluids, and 

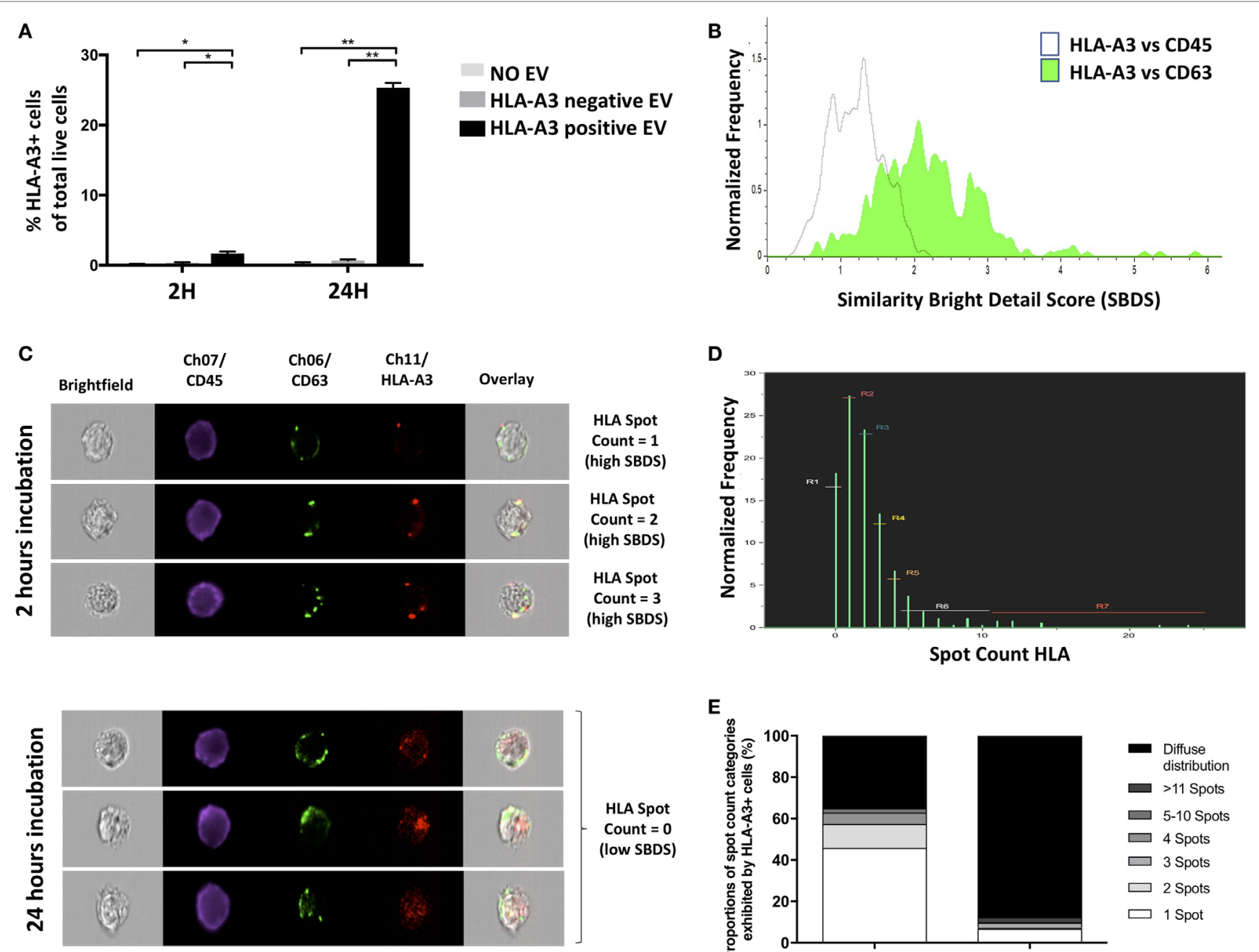

\section{E}

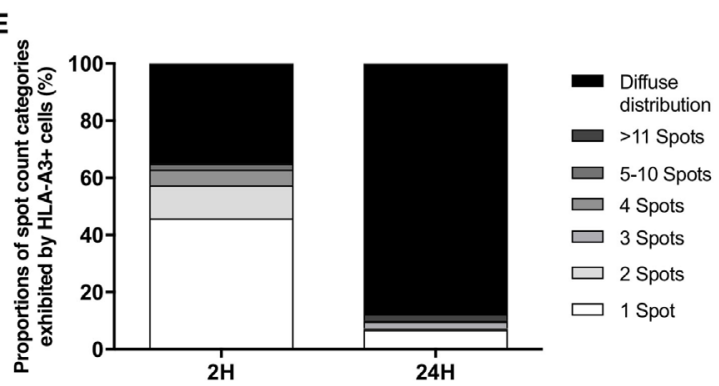

FIGURE 4 | Target-cell analysis determines exosome-mediated molecular transfer (Application 3). (A) Percentage of HLA-A3-positive cells detected by ISx following coculture of CD14+ cells from HLA-A3-negative individual with extracellular vesicles from HLA-A3 positive individual. Data represented as mean \pm SD of two separate experiments. ${ }^{*} P<0.05$ and ${ }^{*} P<0.01$. (B) Representative histogram showing higher colocalization of fluorescently labeled HLA-A3 with CD63 as compared to CD45, as ascertained by the IDEAS ${ }^{\circledR}$ Similarity Bright Detail Score (SBDS). (C) Representative ISx images of single cells following $2 \mathrm{~h}$ coculture exhibiting discrete HLA-A3 spots and with high HLA-A3/CD63 SBDS scores (left panels), contrasted with images of cells following 24 h coculture exhibiting diffuse HLA-A3 fluorescence and with low HLA-A3/CD63 SBDS scores; suggesting incorporation and diffusion of exosome-derived HLA molecules over time. (D) Representative histogram showing the normalized frequencies of HLA-A3 positive cells exhibiting a given number of HLA-A3 "spots," as ascertained by the IDEAS ${ }^{\circledR}$ Spot Count feature, following $2 \mathrm{~h}$ EV coculture. (E) Summary of proportions of HLA-A3 positive cells exhibiting a given number of HLA-A3 spots, following either 2 or $24 \mathrm{~h}$ EV coculture. Data presented as the mean of two separate experiments.

so on. Rather, it is to be reminded that this variation exists, and to be mindful of the need for subset characterization in a number of contexts. We suggest iFCM is a convenient way to achieve this also. Examples of the instances where sEV subtype profiling may be needed include: (a) to ensure consistency of isolation from a given sample, and so achieve consistency in any analyses performed thereafter; (b) to assist standardization of isolation across different researchers, permitting collaboration or replication; (c) enable interpretations of downstream isolate analysis in terms of the subtype profile; and (d) to provide quantitative confirmation of the presence of exosomes in conjunction with current methods (TEM, Western, NTA). In addition, such analysis could guide the choice of method most appropriate for the requirements of selected downstream analyses. For instance, our data might suggest that if a limited volume of plasma is available and exosomal miRNA profiling is desired, UC may not be the best option due to the low quantity of exosomes recovered. If a larger volume of plasma is available, however, UC may be preferred for the greater enrichment of exosomes achieved. Of course, considerations such as the co-isolation of proteins are important in this context.

Numerous studies purporting to be investigating circulating exosomes, performed predominantly in the pursuit of biomarkers of disease, continue to apply analytic techniques ill-suited to the study of exosomes. The use of flow cytometry platforms incapable of accurately resolving objects of exosomal size is one concern, while the failure to narrow investigations specifically 
to exosomes through the use of appropriate markers is another. An assumption that the "exosome" isolation technique employed purifies exosomes, can lead to erroneous conclusions regarding what in fact is the analysis of a heterogeneous pool of sEV of divergent functional properties. We demonstrate that iFCM coupled with subset-specific markers can be applied to circulating, plasma-derived exosome characterization in order to achieve reliable exosomal-origin determination and functional assessments. Multiparametric analysis can provide insights into the immune function of exosomes identified in this way. This approach should be applicable to the gamut of biofluids, across species, or with a focus on any particular sEV subtype. Due to sEVs' small size, however, the question of whether steric hindrance may affect the number of different antibodies that can be used in labeling remains to be investigated, and so the design of large panels should be approached with caution and will require extensive validation. We have shown that "unprocessed" samples (PPP) can also be used, thereby circumventing adverse impacts of isolation procedures. However, the ISx fluidics requires that small sample volumes are run, and that these are run slowly. Thus, samples need to be sufficiently concentrated if long acquisition times are to be avoided.

The fluidic control of ISx coupled with the presence of continuously running speed beads enable cell/particle enumeration using the "objects per mL" feature within the IDEAS ${ }^{\circledR}$. Our data show this feature to corroborate NTA data in comparisons of the relative concentrations of EVs present in isolates derived by different methods. However, the two approaches were very different in the absolute number of EVs by some orders of magnitude. Enumeration of EVs is notoriously challenging and has yielded widely divergent results (44). A number of platforms are currently in use for EV enumeration, with NTA being among the most commonly applied. Each method has its own merits and drawback, and NTA also suffers from the latter. The device is unable to distinguish vesicles from non-vesicular "debris" which by ISx would be identified and excluded by its CFDA-SE-negativity. Inter-user and inter-experiment variability is also a known concern with NTA, particularly among inexperienced users, though steps are being taken to improve this (45-48). The merits of FCM for particle enumeration over other techniques such as NTA are beyond our scope and described elsewhere $(14,49,50)$. What is noteworthy however, is the capacity for iFCM to enumerate sEV subsets specifically, rather than vesicles taken together, or indeed merely the vesicle-sized particles measured by some other techniques. More work is needed for a standardized approach to particle enumeration to be agreed upon.

The use of ISx is also associated with some drawbacks. The particularly large file sizes produced require appropriate storage and processing capacity on any computers used for data analysis, and analyzing data be extremely time intensive. Each alteration to a compensation matrix once acquisition has been performed, requires large files to be re-processed and re-opened. The merging of large files for merged graphical analyses is also laborious. While the setting up of Masks and Feature functions requires the development of some familiarity with the analysis software, the provided Wizards can greatly assist in this process and once satisfactory set-up is achieved, files can be easily batch processed.

\section{CONCLUSION}

Taken together, the data and methods presented offer solutions to two important problems facing EV research. The first relates to the necessity for the subtype characterization of sEV isolates. Without this, the field will continue to produce apparently contradictory EV functional assessments, with comparison or collaboration between individuals or across centers remaining difficult. The second is the need for a validated approach for the multiparametric analysis of bona fide circulating exosomes. Here, we show this to be possible by coupling our emerging understanding of subset-specific markers with the use of advanced iFCM. In this synthesis of our experience characterizing sEVs, we hope to offer a reference to guide EV researchers, perhaps new to the field, in the necessary subset-analysis of isolates, in the multiparametric profiling of exosomes or other sEV subsets, and in the assessment of exosome-mediated molecular transfer. In doing so, we hope to support ongoing research into the function and clinical utility of EVs.

\section{ETHICS STATEMENT}

The study was approved by the North of Scotland Research Ethics Committee (REC Ref: 15/NS/0062). All subjects gave written informed consent in accordance with the Declaration of Helsinki.

\section{AUTHOR CONTRIBUTIONS}

SM, GB, FD, AS-F, and MM-L designed the study and developed methodology. SM and GW collected samples. SM, GB, and MM performed and analyzed experiments. SM, MM-L, and AS-F prepared the manuscript. MM-L and AS-F supervised the project.

\section{ACKNOWLEDGMENTS}

We would like to thank all the subjects and patients who volunteered for this study. The authors would like to acknowledge in particular the support of Prabhjoat Chana technical aspects of ISx use, and expertise in the use of IDEAS ${ }^{\circledR}$; Professor Khuloud Al-Jamal for her kind gift of fluorescently labeled liposomes; and Gema Vizcay and the Centre for Ultrastructural Imaging KCL, for her generous support with exosomal TEM imaging techniques. This study was supported by the King's Medical Research Trust, by the MRC Centre for Transplantation (J006742/1), and by the National Institute for Health Research Biomedical Research Centre based at Guy's and St. Thomas' National Health Service Foundation Trust and King's College London.

\section{SUPPLEMENTARY MATERIAL}

The Supplementary Material for this article can be found online at https://www.frontiersin.org/articles/10.3389/fimmu.2018.01583/ full\#supplementary-material. 


\section{REFERENCES}

1. Wen C, Seeger RC, Fabbri M, Wang L, Wayne AS, Jong AY. Biological roles and potential applications of immune cell-derived extracellular vesicles. J Extracell Vesicles (2017) 6:1-19. doi:10.1080/20013078.2017.1400370

2. Xu R, Greening DW, Zhu H-J, Takahashi N, Simpson RJ. Extracellular vesicle isolation and characterization: toward clinical application. J Clin Invest (2016) 126:1152-62. doi:10.1172/JCI81129

3. Pitt JM, Kroemer G, Zitvogel L. Extracellular vesicles: masters of intercellular communication and potential clinical interventions. JClin Invest (2016) 126:1139-43. doi:10.1172/JCI87316

4. Yáñez-Mó M, Siljander PRM, Andreu Z, Bedina Zavec A, Borràs FE, Buzás EI, et al. Biological properties of extracellular vesicles and their physiological functions. J Extracell Vesicles (2015) 4:27066-27060. doi:10.3402/jev.v4.27066

5. Robbins PD, Dorronsoro A, Booker CN. Regulation of chronic inflammatory and immune processes by extracellular vesicles. JClin Invest (2016) 126:1173-80. doi:10.1172/JCI81131

6. Robbins PD, Morelli AE. Regulation of immune responses by extracellular vesicles. Nat Rev Immunol (2014) 14(3):195-208. doi:10.1038/nri3622

7. Kowal J, Arras G, Colombo M, Jouve M, Morath JP, Primdal-Bengtson B, et al. Proteomic comparison defines novel markers to characterize heterogeneous populations of extracellular vesicle subtypes. Proc Natl Acad Sci U S A (2016) 113:E968-77. doi:10.1073/pnas.1521230113

8. Baek R, Søndergaard EKL, Varming K, Jørgensen MM. The impact of various preanalytical treatments on the phenotype of small extracellular vesicles in blood analyzed by protein microarray. J Immunol Methods (2016) 438:11-20. doi:10.1016/j.jim.2016.08.007

9. Tkach M, Kowal J, Théry C. Why the need and how to approach the functional diversity of extracellular vesicles. Philos Trans R Soc B (2017) 373: 20160479-20160479. doi:10.1098/rstb.2016.0479

10. Bobrie A, Colombo M, Krumeich S, Raposo G, Théry C. Diverse subpopulations of vesicles secreted by different intracellular mechanisms are present in exosome preparations obtained by differential ultracentrifugation. J Extracell Vesicles (2017) 1:18397-18311. doi:10.3402/jev.v1i0.18397

11. Andreu Z, Yáñez-Mó M. Tetraspanins in extracellular vesicle formation and function. Front Immunol (2014) 5:442. doi:10.3389/fimmu.2014.00442

12. Chandler WL. Measurement of microvesicle levels in human blood using flow cytometry. Cytometry B Clin Cytom (2016) 90(4):326-36. doi:10.1002/ cyto.b. 21343

13. Nolan JP. Flow cytometry of extracellular vesicles: potential, pitfalls, and prospects. Curr Protoc Cytom (2015) 73:1-16. doi:10.1002/0471142956. cy1314s73

14. Erdbrügger U, Rudy CK, Etter M, Dryden KA, Yeager M, Klibanov AL, et al. Imaging flow cytometry elucidates limitations of microparticle analysis by conventional flow cytometry. Cytometry (2014) 85:756-70. doi:10.1002/cyto. a.22494

15. Erdbrügger U, Lannigan J. Analytical challenges of extracellular vesicle detection: a comparison of different techniques. Cytometry (2015) 89:123-34. doi:10.1002/cyto.a.22795

16. Arraud N, Linares R, Tan S, Gounou C, Pasquet JM, Mornet S, et al. Extracellular vesicles from blood plasma: determination of their morphology, size, phenotype and concentration. J Thromb Haemost (2014) 12:614-27. doi: $10.1111 /$ jth. 12554

17. Headland SE, Jones HR, D'Sa ASV, Perretti M, Norling LV. Cutting-edge analysis of extracellular microparticles using imageStream ${ }^{\mathrm{X}}$ imaging flow cytometry. Sci Rep (2014) 4:5237. doi:10.1038/srep05237

18. Lannigan J, Erdbruegger U. Imaging flow cytometry for the characterization of extracellular vesicles. Methods (2017) 112:55-67. doi:10.1016/j. ymeth.2016.09.018

19. Witwer KW, Buzás EI, Bemis LT, Bora A, Lässer C, Lötvall J, et al. Standardization of sample collection, isolation and analysis methods in extracellular vesicle research. J Extracell Vesicles (2013) 2:18389-18325. doi:10.3402/jev.v2i0.20360

20. Welton JL, Webber JP, Botos L-A, Jones M, Clayton A. Ready-made chromatography columns for extracellular vesicle isolation from plasma. J Extracell Vesicles (2015) 4:23430-9. doi:10.3402/jev.v4.27269

21. de Menezes-Neto A, Sáez MJF, Lozano-Ramos I, Segui-Barber J, Martin-Jaular L, Estanyol Ullate JM, et al. Size-exclusion chromatography as a stand-alone methodology identifies novel markers in mass spectrometry analyses of plasma-derived vesicles from healthy individuals. J Extracell Vesicles (2015) 4:569-514. doi:10.3402/jev.v4.27378

22. Lobb RJ, Becker M, Wen Wen S, Wong CSF, Wiegmans AP, Leimgruber A, et al. Optimized exosome isolation protocol for cell culture supernatant and human plasma. JExtracell Vesicles (2015) 4:211-211. doi:10.3402/jev.v4. 27031

23. Morales-Kastresana A, Telford B, Musich TA, McKinnon K, Clayborne C, Braig Z, et al. Labeling extracellular vesicles for nanoscale flow cytometry. $\mathrm{Sci}$ Rep (2017) 7:1-10. doi:10.1038/s41598-017-01731-2

24. Pospichalova V, Svoboda J, Dave Z, Kotrbova A, Kaiser K, Klemova D, et al. Simplified protocol for flow cytometry analysis of fluorescently labeled exosomes and microvesicles using dedicated flow cytometer. J Extracell Vesicles (2015) 4:75-15. doi:10.3402/jev.v4.25530

25. Hodgins NO, Al-Jamal WT, Wang JT-W, Parente-Pereira AC, Liu M, Maher J, et al. In vitro potency, in vitro and in vivo efficacy of liposomal alendronate in combination with $\gamma \delta \mathrm{T}$ cell immunotherapy in mice. J Control Release (2016) 241:229-41. doi:10.1016/j.jconrel.2016.09.023

26. Jenner D, Ducker C, Clark G, Prior J, Rowland CA. Using multispectral imaging flow cytometry to assess an in vitro intracellular Burkholderia thailandensis infection model. Cytometry (2016) 89:328-37. doi:10.1002/cyto.a. 22809

27. Pugsley HR. Quantifying autophagy: measuring LC3 puncta and autolysosome formation in cells using multispectral imaging flow cytometry. Methods (2017) 112:147-56. doi:10.1016/j.ymeth.2016.05.022

28. Zuba-Surma EK, Kucia M, Abdel-Latif A, Lillard JW Jr, Ratajczak MZ. The ImageStream system: a key step to a new era in imaging. Folia Histochem Cytobiol (2007) 45(4):279-90.

29. Hennies CM, Lehn MA, Janssen EM. Quantitating MHC class II trafficking in primary dendritic cells using imaging flow cytometry. J Immunol Methods (2015) 423:18-28. doi:10.1016/j.jim.2015.04.023

30. Beum PV, Lindorfer MA, Hall BE, George TC, Frost K, Morrissey PJ, et al. Quantitative analysis of protein co-localization on B cells opsonized with rituximab and complement using the ImageStream multispectral imaging flow cytometer. J Immunol Methods (2006) 317:90-9. doi:10.1016/j.jim.2006.09.012

31. Gardiner C, Shaw M, Hole P, Smith J, Tannetta D, Redman CW, et al. Measurement of refractive index by nanoparticle tracking analysis reveals heterogeneity in extracellular vesicles. J Extracell Vesicles (2014) 3:25361-6. doi:10.3402/jev.v3.25361

32. Van Der Pol E, Coumans FAW, Grootemaat AE, Gardiner C, Sargent IL, Harrison P, et al. Particle size distribution of exosomes and microvesicles determined by transmission electron microscopy, flow cytometry, nanoparticle tracking analysis, and resistive pulse sensing. J Thromb Haemost (2014) 12:1182-92. doi:10.1111/jth.12602

33. Headland SE, Jones HR, Norling LV, Kim A, Souza PR, Corsiero E, et al. Neutrophil-derived microvesicles enter cartilage and protect the joint in inflammatory arthritis. Sci Transl Med (2015) 7:ra190-315. doi:10.1126/ scitranslmed.aac5608

34. Baranyai T, Herczeg K, Onódi Z, Voszka I, Módos K, Marton N, et al. Isolation of exosomes from blood plasma: qualitative and quantitative comparison of ultracentrifugation and size exclusion chromatography methods. PLoS One (2015) 10:e0145686. doi:10.1371/journal.pone.0145686

35. Helwa I, Cai J, Drewry MD, Zimmerman A, Dinkins MB, Khaled ML, et al. A comparative study of serum exosome isolation using differential ultracentrifugation and three commercial reagents. PLoS One (2017) 12:e0170628. doi:10.1371/journal.pone.0170628

36. Ono Y, Perez-Gutierrez A, Nakao T, Dai H, Camirand G, Yoshida O, et al. Graft-infiltrating PD-L $1^{\text {hi }}$ cross-dressed dendritic cells regulate anti-donor $\mathrm{T}$ cell responses in mouse liver transplant tolerance. Hepatology (2018) 67(4):1499-515. doi:10.1002/hep.29529

37. Pols MS, Klumperman J. Trafficking and function of the tetraspanin CD63. Exp Cell Res (2009) 315:1584-92. doi:10.1016/j.yexcr.2008.09.020

38. Ortyn WE, Hall BE, George TC, Frost K, Basiji DA, Perry DJ, et al. Sensitivity measurement and compensation in spectral imaging. Cytometry (2006) 69A:852-62. doi:10.1002/cyto.a.20306

39. Stoner SA, Duggan E, Condello D, Guerrero A, Turk JR, Narayanan PK, et al High sensitivity flow cytometry of membrane vesicles. Cytometry (2015) 89:196-206. doi:10.1002/cyto.a.22787 
40. Welsh JA, Holloway JA, Wilkinson JS, Englyst NA. Extracellular vesicle flow cytometry analysis and standardization. Front Cell Dev Biol (2017) 5:78. doi:10.3389/fcell.2017.00078

41. Tang Y-T, Huang Y-Y, Zheng L, Qin S-H, Xu X-P, An T-X, et al. Comparison of isolation methods of exosomes and exosomal RNA from cell culture medium and serum. Int J Mol Med (2017) 40:834-44. doi:10.3892/ijmm.2017.3080

42. Lacroix R, Judicone C, Poncelet P, Robert S, Arnaid L, Sampol J, et al. Impact of pre-analytical parameters on the measurement of circulating microparticles: towards standardization of protocol. J Thromb Haemost (2012) 10:437-46. doi:10.1111/j.1538-7836.2011.04610.x

43. Tkach M, Théry C. Communication by extracellular vesicles: where we are and where we need to go. Cell (2016) 164:1226-32. doi:10.1016/j.cell.2016. 01.043

44. Arraud N, Gounou C, Turpin D, Brisson AR. Fluorescence triggering: a general strategy for enumerating and phenotyping extracellular vesicles by flow cytometry. Cytometry (2015) 89:184-95. doi:10.1002/cyto.a.22669

45. Vestad B, Llorente A, Neurauter A, Phuyal S, Kierulf B, Kierulf P, et al. Size and concentration analyses of extracellular vesicles by nanoparticle tracking analysis: a variation study. J Extracell Vesicles (2017) 6:1-11. doi:10.1080/ 20013078.2017.1344087

46. Gardiner C, Ferreira YJ, Dragovic RA, Redman CWG, Sargent IL. Extracellular vesicle sizing and enumeration by nanoparticle tracking analysis. J Extracell Vesicles (2013) 2:19671-19611. doi:10.3402/jev.v2i0.19671

47. Filipe V, Hawe A, Jiskoot W. Critical evaluation of nanoparticle tracking analysis (NTA) by NanoSight for the measurement of nanoparticles and protein aggregates. Pharm Res (2010) 27:796-810. doi:10.1007/s11095-010-0073-2
48. Parsons MEM, McParland D, Szklanna PB, Guang MHZ, O'Connell K, O'Connor $\mathrm{HD}$, et al. A protocol for improved precision and increased confidence in nanoparticle tracking analysis concentration measurements between 50 and $120 \mathrm{~nm}$ in biological fluids. Front Cardiovasc Med (2017) 4:68. doi:10.3389/fcvm.2017.00068

49. Pasalic L, Williams R, Siupa A, Campbell H, Henderson MJ, Chen VMY. Enumeration of extracellular vesicles by a new improved flow cytometric method is comparable to fluorescence mode nanoparticle tracking analysis. Nanomedicine (2016) 12:977-86. doi:10.1016/j.nano.2015.12.370

50. Maas SLN, de Vrij J, van der Vlist EJ, Geragousian B, van Bloois L, Mastrobattista E, et al. Possibilities and limitations of current technologies for quantification of biological extracellular vesicles and synthetic mimics. J Control Release (2015) 200:87-96. doi:10.1016/j.jconrel.2014.12.041

Conflict of Interest Statement: The authors declare that the research was conducted in the absence of any commercial or financial relationships that could be construed as a potential conflict of interest.

Copyright (c) 2018 Mastoridis, Bertolino, Whitehouse, Dazzi, Sanchez-Fueyo and Martinez-Llordella. This is an open-access article distributed under the terms of the Creative Commons Attribution License (CC BY). The use, distribution or reproduction in other forums is permitted, provided the original author(s) and the copyright owner(s) are credited and that the original publication in this journal is cited, in accordance with accepted academic practice. No use, distribution or reproduction is permitted which does not comply with these terms. 